\title{
Improvement in the Wear Resistance of Brass Impeller of Slurry Pump used in Variation of Operating Speeds
}

\author{
Ahmed M.M. EI-Bahloul \\ Production Engineering and \\ Mechanical Design \\ Department, \\ Faculty of Engineering \\ University of Mansoura, Egypt
}

\author{
. M.Samuel \\ Production Engineering and \\ Mechanical Design \\ Department, \\ Faculty of Engineering \\ University of Mansoura, \\ Egypt
}

\author{
Mazin.K.I. AL-Mashhadani \\ Ministry of Science and \\ Technology, \\ Iraq
}

\begin{abstract}
Erosion wear is recognized as an engineering problem for Centrifugal slurry pumps it plays an important role in design and operation of slurry transportation systems. In most slurry pump applications the monetary loss due to pump failure consists of loss of production rather than the cost of the pump, since the process must be shut down when the pump fails. If the erosion rate can be estimated, the pump's impeller can be changed out in time, during scheduled maintenance, The present work reports experimental investigations on dependence of erosion wear of brass alloys in sand - water slurry. Systematic study on the failing impeller of a slurry pump used in irrigation has been carried out by means of scanning electron microscopy in order to clarify the failure mechanisms. The results show .The weight loss of the impeller due to the material removal from the impeller as result of erosion wear. In this investigation, experiments were carried out to evaluate the wear resistance using wear test rig designed and manufactured for this purpose. Three specimens of different brass alloys were used, namely (CuZn2Ge-CuZn4Ge-CuZn6Ge) Discs manufactured from. The accumulated loss of weight was measured and the wear rates were determined for each Case material at constant normal test load of $20 \mathrm{~kg}$ and time24hr. Number of revolutions of tests equals (1000-1500-2000) r.p.m. were selected for the three test materials respectively during wear tests .The accumulated loss of weight was measured as a function of the number of revolutions. Wear rate was calculated. The results show Wear rate for material B3 $(\mathrm{CuZn} 6 \mathrm{Ge})$ is less than that of the wear rate for B2 $(\mathrm{CuZn} 4 \mathrm{Ge}), \mathrm{B} 1(\mathrm{CuZn} 2 \mathrm{Ge})$ under the same testing conditions at (1000-1450-2000) r. p. m.
\end{abstract}

Keywords: Brass, Structures, wear test, Wear rate, hardness,

\section{INTRODUCTION}

Pumps play a key role in irrigation and moving and lifting fluids in general to high areas. A mechanical equipment lowly fluid lift from a low level to high level considered pumps Calendar Irrigation of very necessary and important equipment which are used in a wide areas and there was a need to study so that we can choose the appropriate pump for a particular performance as well as the possibility to run different speeds And the impact on its performance in use irrigation pumps efficiently and tak advantage of the increase Productivity the Iraqi farm[1] Erosive Wear is a phenomenon related to material removal from a surface due to interaction with a mating surface. Wear is a common industrial problem, leading to frequent maintenance and replacement of components, and loss associated with production or availability, which also carries economic penalties. In addition, wear may also decrease the operating efficiencies [2]. Wear can generally be divided into two main types that are mechanical wear and chemical wear [3]. It is generally held that the most common types of wear mechanism are the adhesive wear, abrasive wear, cavitation wear, corrosive wear, erosive wear, fatigue wear and fretting wear. Infect, more than a single mechanism can occur at the same time [4].However, there is always a primary mechanism that determines the material removal rate. Impellers wear rate in a slurry pump is an important factor. Slurry pumps are used almost universally to move mixtures of abrasive solid sand liquids. Pump useful life in these applications can range from a few weeks to a few years depending on the type of slurries handled. Major wet end components of a centrifugal slurry pump including impeller wear occurs largely as a result of erosion [5].The term "erosive wear" refers to an unspecified number of wear mechanisms which occur when relatively small particles impact against mechanical components The major type of erosion wear in a slurry pump impeller is due to the Particle impingement.[6].Where the particles impact at angles substantially normal to the surface. The predominant type of wear in centrifugal pump impeller is sliding-bed erosion whereas erosion wear is caused by impact of liquid or solid particles on the solid surface. The main cause of the erosion wear is impact [7]. Particles impacting directly onto a surface can generate very high specific contact pressures. The actual value of the contact stress depends on the particle velocity, the mass, and the particle shape. Particles with sharp edges wear the surface faster because of their smaller contact area (and thus higher stresses). Material is removed by a process of cutting and/or pouching.[8] Pouching Erosion wear: is a two stage process involving localized plastic deformation of the surface from rounded particle impacts [9].Cutting Erosion wear: occurs when the particles are very sharp and interaction with the material surface causes a micromachining action [10].Deformation and Cracking: When cyclic forces are applied to parts found in the pump, a crack may appear over a period of time, in a target surface. Deformation and Cracking can occur on a surface, even though the loading produces stresses that are far less than the tensile strength of the material. after the crack has been introduced into the surface. the crack may grow with each cyclic loading until the surface finally Deformation and Cracking[11].Elemental analysis of the failed impeller blades, as well as the middle of blades, shows that the 
base alloy used for this impeller is Brass. Physical examinations, that the leading edge of the impeller had undergone active corrosion with rough surface and visible pit like features. Chemical analysis of the blade surface at various locations indicated at the surface. This confirms that the erosion wear of surface has occurred on these alloys in the environment to which this impeller was exposed. To verify further the effect of velocity on the erosion rate of an impeller, simulated erosive wear tests were performed on a rotating disk on disk with of tested materials used in this investigation and samples from the failing impeller. Three speeds were used. The first is (1000) $\mathrm{r}$. p. $m$ and the second is (1450) r. p. m. The third (2000)

\section{Experimental Work}

\subsection{Test Specimens}

2.1.1. Materials: used in this investigation for impeller pump production in are as follows: (B1(CuZn2Ge), (B2(CuZn4Ge,(B3(CuZn6Ge). The chemical composition for materials used in this investigation is given in table (1).

\subsubsection{Specimen Dimensions:}

Specimens were prepared from Base metal $(\mathrm{CuZn} 2 \mathrm{Ge})$, (CuZn4Ge), (CuZn6Ge), for wear test, Chemical composition of alloys as Table (1) microstructure and hardness.

\subsubsection{Test specimens for wear:}

Specimens were prepared from Base metal (brass)and $(\mathrm{CuZn} 2 \mathrm{Ge}),(\mathrm{CuZn} 4 \mathrm{Ge}),(\mathrm{CuZn} 6 \mathrm{Ge}), 80 \mathrm{~mm}$ diameter \& $15 \mathrm{~mm}$ length, shown in figure $(1, \mathrm{a})$, which were turned ,shown in figure $(1, \mathrm{~b})$, In this work 24 discs were used , 6 discs from each tested materials divided into 4 groups ;(2) discs for each group follows: shown in Table(2).it used speed (1000,1450,2000), r. p. m

(a)-Cu-Zn (Ge 2\%, 4\%,6\%) 18 discs .

(b)-Base metal (brass) of (6) discs.

\begin{tabular}{|c|c|c|c|c|c|c|}
\hline No. & $\mathrm{Zn} \%$ & $\begin{array}{l}\text { Ge } \\
\%\end{array}$ & $\mathrm{Fe} \%$ & P\% & S\% & $\begin{array}{l}\mathrm{Cu} \\
\%\end{array}$ \\
\hline B1 & $\begin{array}{l}29.98 \\
9\end{array}$ & $\begin{array}{l}2.01 \\
7\end{array}$ & $\begin{array}{l}0.001 \\
8\end{array}$ & 0.006 & $\begin{array}{l}0.00 \\
3\end{array}$ & Rem \\
\hline B2 & $\begin{array}{l}29.98 \\
7\end{array}$ & $\begin{array}{l}4.01 \\
4\end{array}$ & 0.002 & 0.001 & $\begin{array}{l}0.00 \\
1\end{array}$ & Rem \\
\hline B3 & $\begin{array}{l}29.97 \\
5\end{array}$ & $\begin{array}{l}6.01 \\
1\end{array}$ & 0.001 & 0.004 & $\begin{array}{l}0.00 \\
2\end{array}$ & Rem \\
\hline $\begin{array}{l}\text { Base } \\
\text { meta } \\
\text { l }\end{array}$ & $\begin{array}{l}29.86 \\
2\end{array}$ & 0.0 & $\begin{array}{l}0.002 \\
2\end{array}$ & $\begin{array}{l}0.001 \\
5\end{array}$ & $\begin{array}{l}0.00 \\
9\end{array}$ & Rem \\
\hline
\end{tabular}

Table (1) Chemical composition

\begin{tabular}{|l|l|l|l|l|}
\hline Speed(r.p.m) & B1(2\%Ge) & B2(4\%Ge) & 33(6\%Ge) & Base metal \\
\hline 1000 ( r.p.m) & B11/B11” & B21/21" & B31/B31” & brass1/brass1" \\
\hline 1450 (r.p.m) & B12/B12” & B22/B22” & B32/32” & brass2/brass2" \\
\hline 2000 (r.p.m) & B13/B13” & B23/B23" & B33/B33" & brass3/brass3" \\
\hline
\end{tabular}

Table (2) Test Specimens Materials for wear

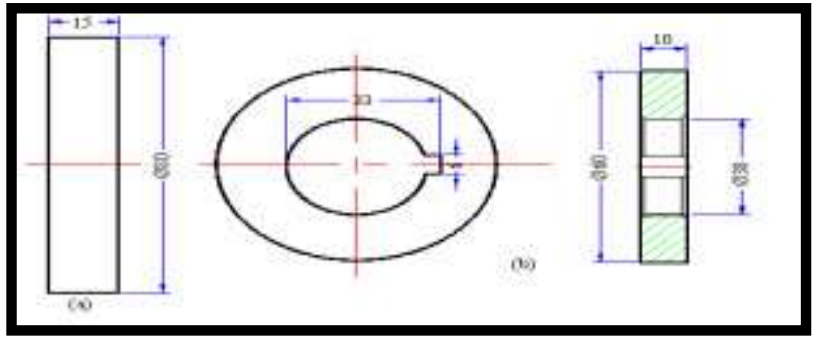

Fig $(1, a, b)$ The shape and dimensions of test discs

\section{Experimental Tests}

\subsection{Wear Test}

Wear is usually measured by one of two methods either by measuring dimensional changes or by weight loss. In this study, wear was estimated and measured by weight loss. Precise digital balance was used. The accuracy of the balance and its capacity is $240 \mathrm{~g}$. By weighting the specimens before and after each run, it was possible to calculate the weight loss due to wear. Thus the accumulated wear as a function of rolling distance can easily be investigated and plotted. From the plots and knowing the density of the material, it was possible to calculate the wear rate of tested materials. [12].

Wear rate $=\mathrm{W} /(\mathrm{P} * \mathrm{~N})$

Where: W: Accumulated weight of removed metal (mg).

$$
\begin{aligned}
& \mathrm{P}: \text { is normal applied load.(kg) } \\
& \mathrm{N} \text { : Total number of revolutions. }
\end{aligned}
$$

\subsection{Wear Testing Machine Disc machine;}

in the two-disc machine, one disc is mounted in a bearing supported by a swinging arm while the other is carried on a rigid bearing. The disc carried by a swinging arm is pressed against the other by a loaded lever. Wear machine manufactured in Gears laboratory in department production engineering and mechanical design: The machine consists of upper casing part (1) cast iron boxes and their housing (3) to carry the bearings support driven discs specimens, lower casing part (2) cast iron boxes and their housing (4) to carry the bearings support driver discs specimens. Bearing housing $(3,4)$ to support the single row deep groove ball bearings (11) $6003 z z$. Driven and drive shaft $(5,6)$ to carry the driven and drive discs (7), spacing sleeve (8) to adjust the distance between the discs and ball bearing , key (9) to fix the discs with the shafts, Snap ring $\Phi 17 \mathrm{~mm}$ (10) to locate the bearing inner race with the shafts, spacer sleeve (12), to adjust the distance between the ball bearing and the driven pulley (13) which fix by key (16) , the washer (14) and the bolt (15) to fixed the pulley. Base (17) Dimensions, $400 * 600 \mathrm{~mm}$, the base is supported on four angles (legs) $50 * 50 * 300 \mathrm{~mm}$ (25). Driving motor (24) three phase, 2 horse powers, 1450 r.p.m and 380 volts was used to drive the machine. 
A calibrated two springs (21) supported by two studs (18) and four bases $(20,22)$ to apply the required load between the drive and driven discs by the tightening nuts (19) to deflect the springs. Two dial gauges measurements range from 0 to $50 \mathrm{~mm}$ and accuracy $0.01 \mathrm{~mm}$ (23) were mounted to measure the deflection of the springs; this deflection represents the exerted load. Figures (2) represent a schematic layout of the machine.

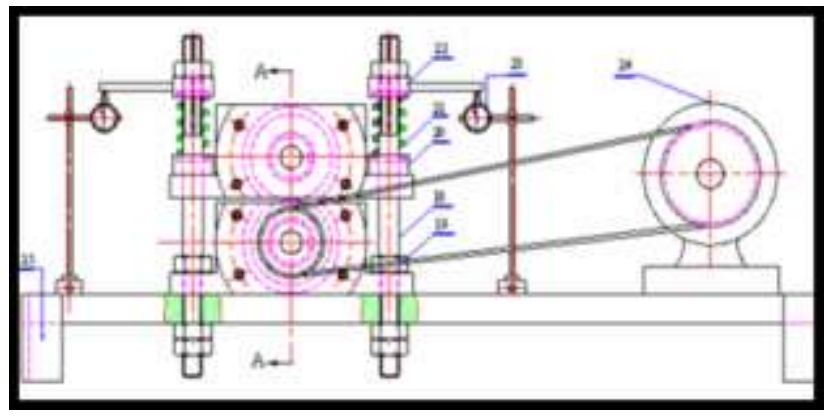

Fig (2) schematic layout of the machine test

\subsection{Calibration Curve of spring: Calibrating} curve for the two springs to apply the required load between the drive and driven rollers is shown in Fig. (3)

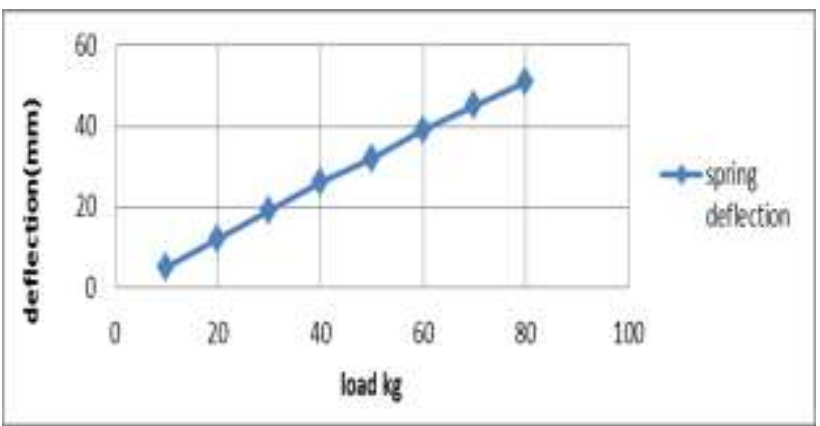

Fig (3) Calibration curve of spring

\subsection{Wear Test Procedure}

The procedure for wear test experiments can be summarized in the following steps: (1) Before each test, the discs were carefully cleaned.(2) Load: Tests were carried out at constant load of $20 \mathrm{~kg}$, the normal loads which were applied through two springs, were maintained constant during the period of test .(3) Speed : Speed designed rollers used for the purpose of converting speeds to obtain the required speeds and by country and energies of the electric motor from to( 1000 1450 - 2000 r.p.m) (4) The machine was stopped at time intervals for weighing the discs. Any loose debris at the surface of the disc specimens were removed by cleaning before weighing the specimens. (5) On restarting the wear tests, the discs were located in their supports in exactly the same orientation as before stopping the tests, in order to ensure that the wear in discs has occurred in the same direction throughout the test.( 6) At the end of the test, the accumulated wear of the discs, expressed in $\mathrm{mg}$. was plotted against the number of revolutions and the wear rate were calculated for each test material.

\section{Results and discussion}

\subsection{Results of hardness test}

Figure(4) represents the relationship between hardness and the percentage by additive of germanium, that the relationship between them is a direct relationship, the hardness of the alloy principally be the result of the solution rigid resulting from the melting of copper in zinc which is less hardness than other alloys, note the increased hardness with the increase in the proportion of germanium and be higher hardness ratio at adding of $6 \%$ germanium is due to increase hardness with increasing the proportion of germanium that this article has a high hardness its presence in the alloy increases the hardness as well as the work of minutes germanium to impede the movement of dislocations, and impeding the plastic deformation of the alloy, leading to increased hardness.

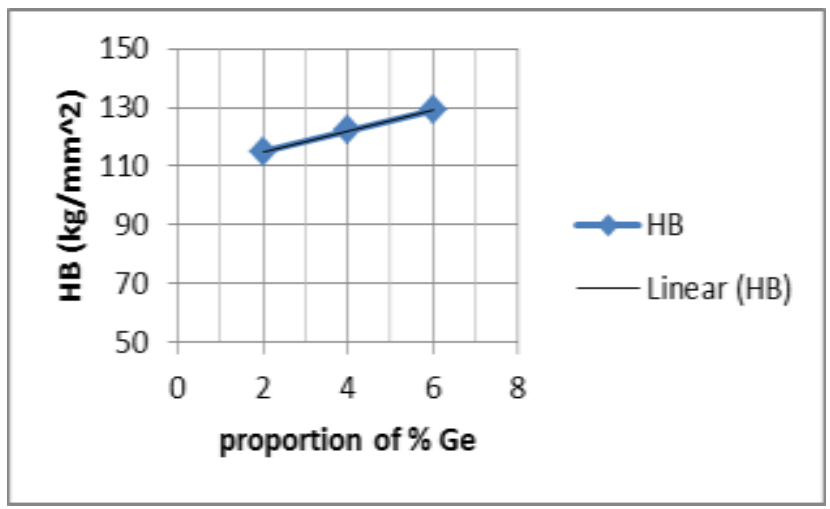

Fig (4) the relationship between hardness and the proportion of germanium.

\subsection{Effect of percentage added germanium on the accumulated loss of weight to driving}

driven: The major type of wear that take place in this experimental work is erosion. The weight loss of the impeller is due to the material removal from the impeller as a result of erosion wear. The entire testing was divided in three phases. The details of all three phases are mentioned below: First Phase: $1000 \mathrm{rpm}$, load 20kg as in Figure (5).Second Phase: $1450 \mathrm{rpm}$, load 20kg as in Figure (6), Third phase: $2000 \mathrm{rpm}$, load $20 \mathrm{~kg}$ as in Figure (7).It was used in load all speeds of 20 $\mathrm{kg}$ and time $24 \mathrm{hr}$. The duration of each test rate three hours. Germanium has been studied weight ratios on the basis alloys and other alloys loss rate when the load $(20 \mathrm{~kg})$ and different speeds $(1000.1450 .2000)$ rpm.the fig $(5,6,7)$ note the relationship between the number of revolutions and weight losing rate where the relationship is directly proportional to each of the basis slug and alloys other and note that the basis slug have a loss larger than the other alloys weight rate and note less loss rate by weight in the alloy (B3_6\%Ge).Any the element increase germanium least the of weight loss rate. This is due to the increase of the proportion of the added germanium element increases the hardness and as described in the hardness test where the hardness is inversely proportional to the rate of weight loss as reduce the (Plastic deformation).In the early stages of the sliding wear rate increase be greater than the closing stages when a high time periods, due to the time period 
increase for the sliding lead increase .In flattening the bumps in both adjacent surfaces are obtained on the surface outcrops in which the proportion of a few, in addition to continuing to slide lead to a declamatory hardening of the surface of the Sample and thus lower metal loss rate. It is noted that the basis slug more affected by a time of sliding of overlapping material where noted that the metal loss rate decreases with the increase of the at germanium $6 \%$ and be less loss rate by weight and this goes back to the role of germanium element to increase the hardness of the sample and to reduce contact between surfaces adjacent and reduce the rate of loss by Weight.

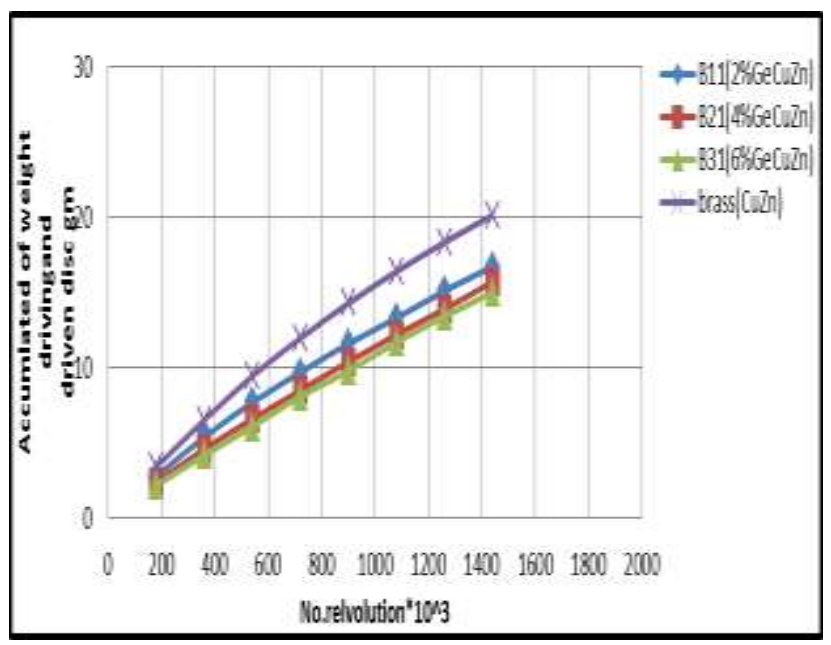

Fig (5) The variation of accumulated loss of weight of driving and driven discs versus the number of revolutions at different materials, (B11/B11”), (B21/B21"), (B31/B31"), at velocity (1000) r. p. m.

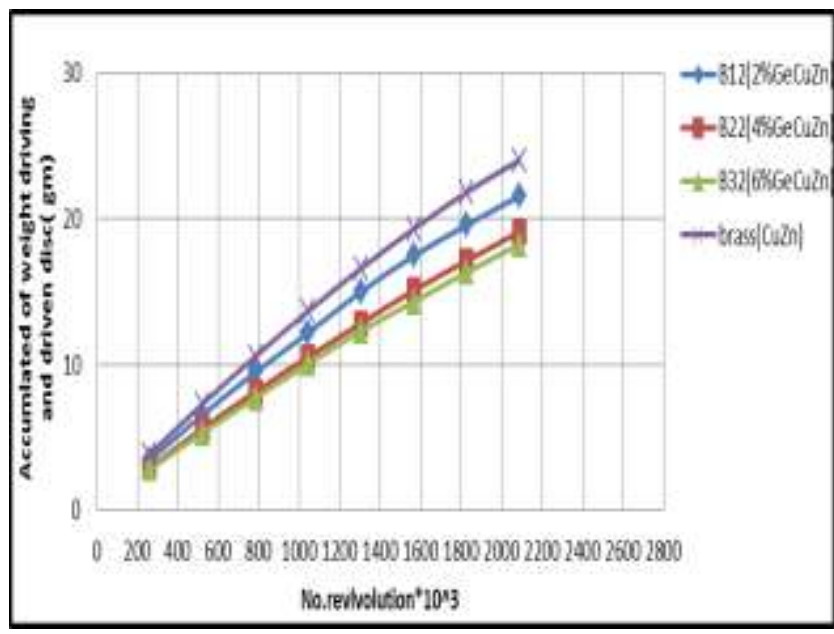

Fig (6)The variation of accumulated loss of weight of driving and driven discs versus the number of revolutions at different materials, (B12/12") (B22/B22") (B32/B32”) at velocity (1450) r. p.m.

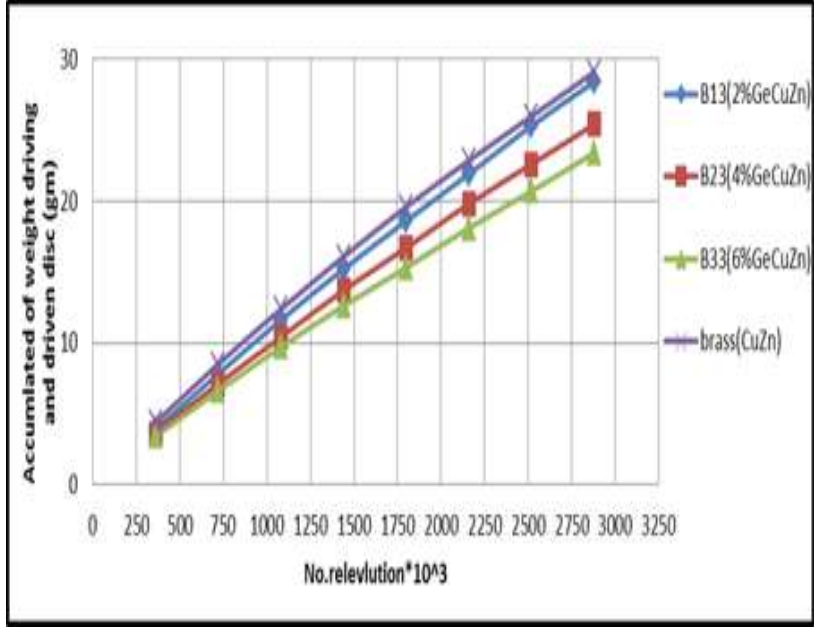

Fig (7) The variation of accumulated loss of weight of driving and driven discs versus the number of revolutions at different materials, (B13/B13")(B23/B23")(B33/B33") at velocity (2000) r. p. m.

\subsection{Effect of percentage of Added Ge\% on}

the wear rate: Effect add percentage of germanium on the wear rate of the basis alloy and other alloys at different speeds (1000-1450-2000) rpm and prove used load $(20 \mathrm{~kg})$ and time. It is the shape (8) note the relationship between the ratios added and the wear rate is where the relationship is inverse of each of the basis alloy and other alloys note that the basis alloy have the wear rate high and note less the rate wear in the alloy (B3_6\% Ge). Any that top element Germanium at wear rate least. This is due to the increase of the proportion of the additives element germanium increases the hardness

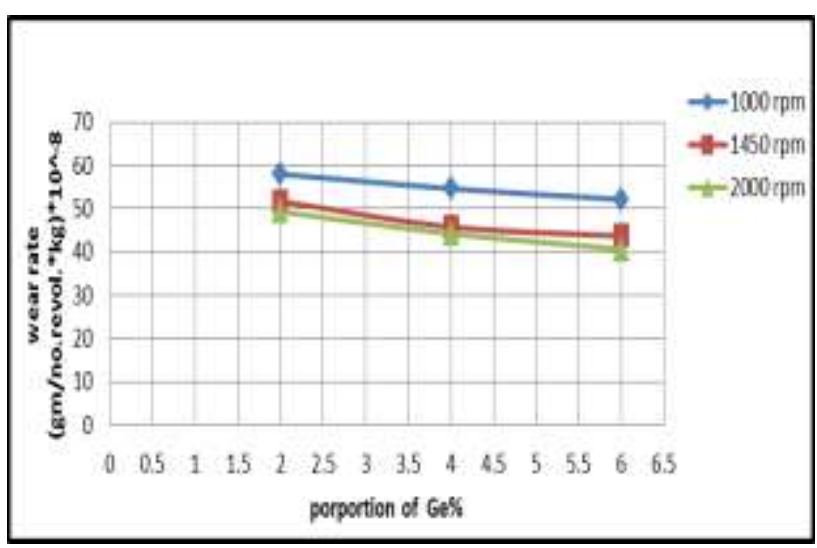

Fig (8) effect of the proportion of germanium on the wear rate at different speed 


\subsection{Effect of speed on the wear rate:}

The effect of speed on the wear of the basis slug with other alloys prove load rate (20kg) and time ( 24 hours.). Where note of the form (9) with low wear rate increase the speed of the basis alloy and other alloys that wear rate Higher when a speed few can be Attributed to obtain partial fusion between the protrusions sliding surface due to the heat seeping from the sample surface at low velocities high and thus the force required to cut the points of contact between the protrusions is greater than the power of the metal interconnection, either low wear rate the speed increase of the slide it may be due to the heat leaking at speed few high slip and so result formed oxide layer on the contact surface which helps to decrease in the rate of wear.

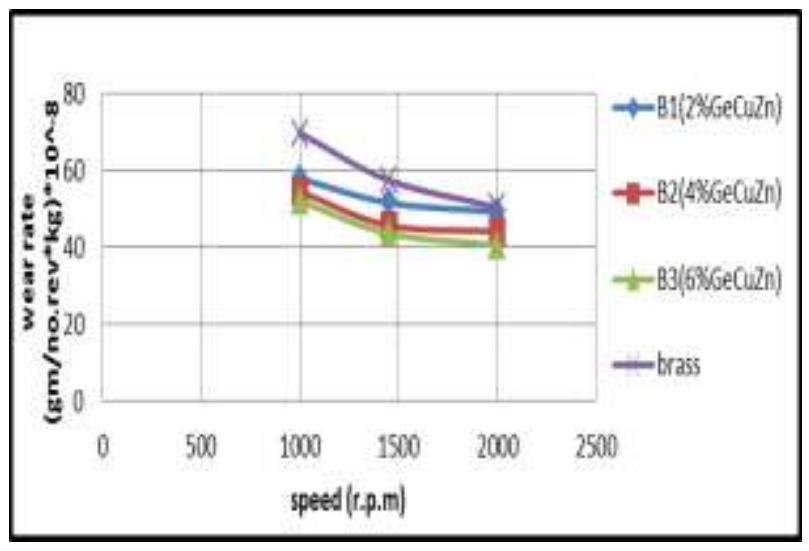

Fig (9) Effect of speed on the wear rate

\subsection{Microstructure test:}

Figure shows (10.a) which pictures the microscopic structure of brass when cast in sand .Which should, if cooled at an equilibrium rate, have shown nothing but a single solid solution. The photograph shows a marked dendritic structure indicating the gradation in composition in the crystals as they formed. If this casting should be annealed for a long time, the structure should become homogeneous. but for the forms (b,c,d),(fig 10) which shows the Microscopic structure of the alloy after the addition of germanium element and different proportions (Ge\%2-6).

The Installation begins dendritic Change and improvement in infrastructure because of the work centers Terms of minutes, and noticed that the element germanium is distributed almost other inside the alloys. We also note that there is a light-colored area, which represent ground which is the solution solid such as copper and zinc.

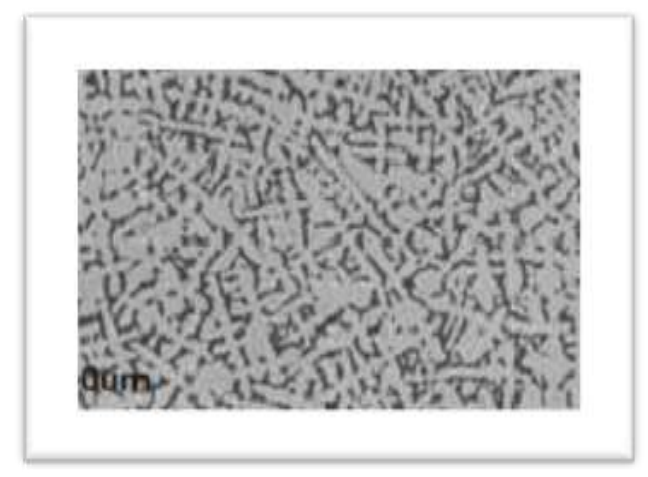

(a)- Brass $(\mathrm{Cu}-\mathrm{Zn}), 100 \mathrm{x}$

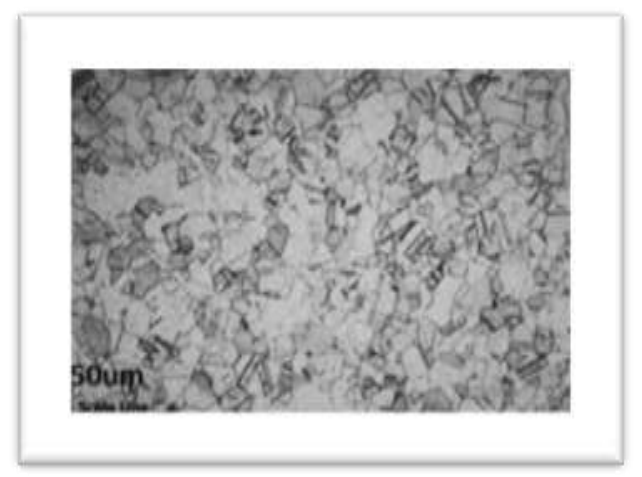

(b) - B1(2\%GeCuZn), 100x

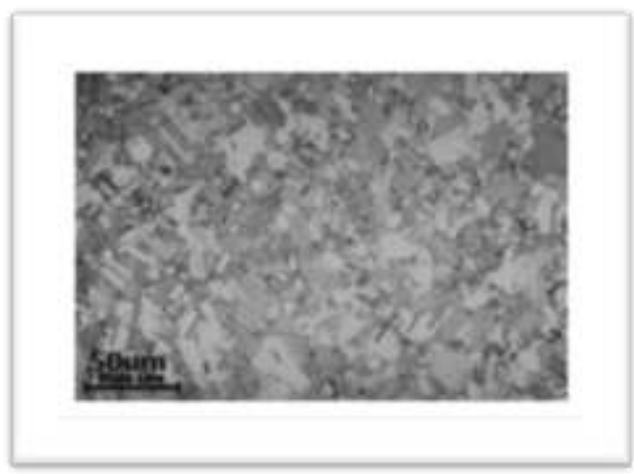

(c)- B2 (4\%GeCuZn), $100 x$

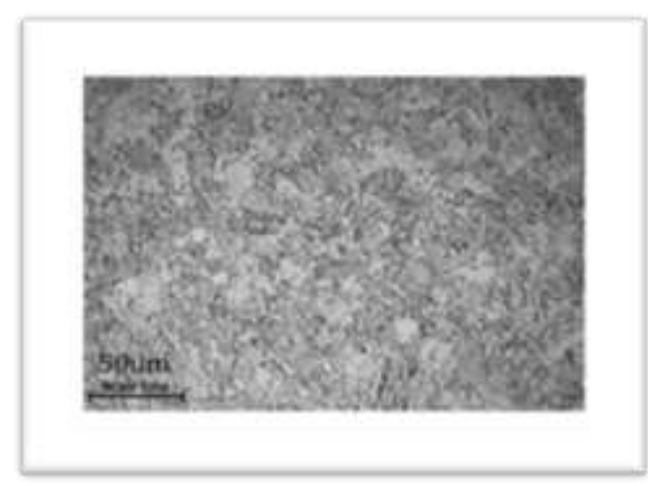

(d) - B3 (6\% GeCuZn), 100x

Fig (10) the microscopic structure of the alloys 


\section{CONCLUSIONS}

1. Wear rate for all materials under investigation decreases with the increase of their case hardness. Minimum wear rate was obtained at hardness $129 \mathrm{HB}$.

2. Wear rate for all tested materials decreases with the increase of germanium. The addition of germanium to reduce the wear the alloys and the wear rate decrease increases with added lineage of germanium element and less wear rate was found when the proportion addition at $6 \% \mathrm{Ge}$.

3. Notes through graphics that increase the element germanium leads to the gradually reduce the rate of wear due to the increase of germanium element which increases the hardness and reduce the wear and tear of the metal.

4. Wear rate for material $\mathrm{B} 3(6 \% \mathrm{GeCu} \mathrm{Zn})$ is less than that of the wear rate for $\mathrm{B} 2(4 \% \mathrm{GeCu} \mathrm{Zn}), \mathrm{B} 1(2 \% \mathrm{GeCu} \mathrm{Zn})$ and Base metal respectively under the same testing conditions at (1000-1450-2000) r.p.m.

5. Wear rate is reduced to basis alloy and other alloys and increase speed

6. Best alloy through the results that have been reached are B3 $(6 \% \mathrm{GeCuZn})$ at different speeds, which gives better resistance to wear and erosion that can through these specifications manufacturing pumps impeller.

7. Through the results obtained $\mathrm{B} 3(6 \% \mathrm{GeCuZn})$ can be designed to pump speeds, which gives impeller erosion rate less, maintenance less and a possible pump to reduce the economic cost and long life impeller.

\section{ACKNOWLEDGEMENTS}

It is hard to begin to put into words how someone took me from a place I can only Describe as being in the dark into a place of light.Prof.Dr.Ahmed-EL-Bahloul,Ball provided that ray of sunshine that enabled me to complete this study. I will be indebted to him always.I would like to express my sincere gratitude. Dr.Miajdi.Samuel for his support and guidance, above and beyond the technical during my research

\section{REFERENCES}

[1].Suhane.A,'Experimental Study on Centrifugal Pump to Determine the Effect of Radial Clearance on Pressure Pulsations, Vibrations and Noise",(IJERA),Vol.2, Issue4, July-August (2012), pp.1823-1829.

[2]. majid.Z.A,mohsin R. and yusof .M.Z,"erosion wear of natural gas pipes due to high velocity jet physical examination and experimental study", jurnal teknology, 56 (Sains \& Kej.) Keluaran Khas (1), Dis. (2011),pp. 1-25.

[3].Aiming.F, Jinming. L. and Ziyun.T. "Failure analysis of the impeller of a slurry pump subjected to corrosive wear", Wear, Vol. 181-183, (1995), pp. 876-82.

[4].Sharma.A. K," numerical study of erosion wear on a Centrifugal slurry pump",MS, mechanical engineering deperment,thapar unversity patila, JUNE (2008),pp.11-14.

[5].Jirout.T,F..I," Study of erosion wear of pitched blade impellers in a solid-liquid suspension", Prosimy cytować jako: Inż. Ap. Chem. (2009), 48, 4, pp. 58-59.

[6].Gnanavelu.A.B,"A geometry independent integrated method to predict erosion wear rates in a slurry environment",Ph.D, University of Leeds, School of Mechanical Engineering, December( 2010),p.13.

[7].Patil. M. S, Deore E. R, Jahagirdar .R. S, Patil.S. V ," Study of the Parameters -Affecting Erosion Wear of Ductile Material in Solid-Liquid Mixture, World Congress on Engineering, U.K,( 2011), Vol III,p.1

[8].dhinda.D.S,"parametric study of erosion wear of solidliquid matrix",Ms,production and industrial engineering,thapar university , june( 2011),p.6.

[9].Kumar.R,"investigation of erosion wear of ductile material with and without coating" ,Ms,production industrial engineering,thapar university ,july (2011),ch1,p.4

[10].www.Wikipedia.com, wear,this page was last modified on 13 may (2014) at 07:40.

[11].Kristoffer.K.,McKee,Forbes.G,Mazhar.I, Entwistle.R and Howard.L.," A review of major centrifugal pump failure modes with application to the water supply and sewerage industries", ICOMS Asset Management Conference, May 16,(2011),PP.8. 